\title{
Global attractors of pinched skew products
}

Glendinning, Paul

2002

MIMS EPrint: 2006.88

Manchester Institute for Mathematical Sciences

School of Mathematics

The University of Manchester

\footnotetext{
Reports available from: http://eprints.maths.manchester.ac.uk/

And by contacting: The MIMS Secretary

School of Mathematics

The University of Manchester

Manchester, M13 9PL, UK
} 


\title{
GLOBAL ATTRACTORS OF PINCHED SKEW PRODUCTS
}

\author{
PAUL GLENDINNING
}

\begin{abstract}
A class of skew products over irrational rotations of the circle is defined which contains some systems which have strange nonchaotic attractors. The global attractor of these systems is characterized: it lies between an upper semi-continuous curve and a lower semi-continuous curve. With additional assumptions on the class of maps considered more detail of the attractor can be given.
\end{abstract}

Mathematics Subject Classification: 58F11

\section{INTRODUCTION}

The theory of quasiperiodically forced systems is still poorly developed compared to the well-established phenomenology associated with periodically forced systems. It has become clear that some remarkable new dynamical features are possible when more than one forcing frequency is used; in particular strange nonchaotic attractors may be observed over a large set of parameter values (Grebogi et al. 1984; Bondeson et al. 1985; Romeiras and Ott 1987). Although the existence of these attractors has been established numerically, there are relatively few rigorous results in the literature and so it is hard to know how best to characterise the invariant sets or the dynamics on these sets. In systems forced at two independent frequencies, the discrete time map obtained by observing the state at times separated by the period of one of the forcing terms will have an angular variable, $x$, which advances by a constant amount at each time step. This corresponds to the advance of the phase associated with the second forcing frequency. These maps are easier to treat both analytically and numerically and have been the source of a rich variety of dynamics (Grebogi et al. 1984; Bondeson et al. 1985; Romeiras and Ott 1987; Pikovsky and Feudel 1994; Feudel et al. 1995; Stark 1997).

To illustrate the idea of strange nonchaotic attractors, Grebogi et al. (1984) introduced the system

$$
x_{n+1}=x_{n}+\omega, y_{n+1}=B \cos 2 \pi x_{n} \tanh y_{n}
$$

where $x \in \mathbb{T}^{1}$ is taken $\bmod 1, \omega=\frac{1}{2}(\sqrt{5}-1)$ and $B>0$. This map is a skew product: the angle $x$ evolves independently of $y$, whilst the evolution of $y$ depends upon both

Date: May 22, 2002. 


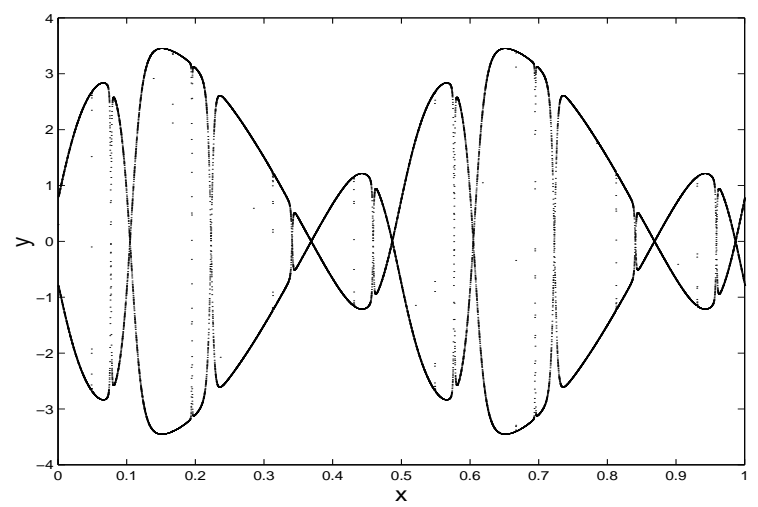

FiguRE 1. The strange nonchaotic attractor of the map (1) with $\omega=$ $(\sqrt{5}-1) / 2$ and $B=3.6$. The horizontal axis is $x$ and the vertical axis is $y .20000$ points on the orbit with initial condition $(0,0.3)$ are plotted. Theoretically, the set in $y>0$ is upper semi-continuous and the set in $y<0$ is lower semi-continuous.

$y$ and $x$. Grebogi et al. (1984) note that the circle $y=0$ is invariant under (1) with stability determined by the Liapunov exponent of $y=0$ in the $y$-direction,

$$
\lambda_{0}=\int_{0}^{1} \log |B \sin 2 \pi x| d x=\log B-\log 2
$$

If $\lambda_{0}<0$ (i.e. $0<B<2$ ) then the circle $y=0$ is locally stable, whilst if $\lambda_{0}>0$ (i.e. $B>2$ ) then the circle $y=0$ is unstable and typical solutions close to $y=0$ move away from this circle. On the other hand, if $x_{n} \in\left\{\frac{1}{4}, \frac{3}{4}\right\}$ then $y_{n+1}=0$ independent of $y_{n}$ and so at least some solutions map to the unstable circle. Moreover, $\left|y_{n}\right|<B$, so solutions are bounded. Hence there is an attractor, and it must contain the unstable set $y=0$. Indeed, for the dense set of $x$ values which are images of the critical set $\left\{0, \frac{1}{2}\right\}$ the only possible value the attractor can take is $y=0$, so the attractor is 'pinched' at this dense set of points. Figure 1 shows a numerical simulation of the attractor for $B>2$ (after Grebogi et al. (1984)). This figure explains why such attractors are called strange: the upper and lower curves must intersect on a set which is dense on the circle $y=0$, so the attractor is geometrically very complicated. Moreover, the Liapunov exponent in the $y$-direction is negative on typical points of the attractor, and so the attractor is nonchaotic. This is an example of a strange nonchaotic attractor.

More recently Keller (1996) has given a general theory for a class of maps which includes this example. However, the maps which are described by this theory are still very special (for example, one of the conditions is that a particular function is convex). For the cases covered by Keller's result, the strange nonchaotic attractor 
is the invariant graph of a measurable function, which is upper semi-continuous (see definitions below) but not continuous. The aim of this note is to extend aspects of Keller's results to a broader class of skew products which we call pinched skew products. In this more general class of maps, the global attractor (Hale 2001) can be described completely, although the full details of the dynamics remain unclear. In particular, although this note was motivated by the study of strange nonchaotic attractors, chaotic attractors are also possible in pinched skew products.

\section{Pinched Skew Products and statement of the main Result}

Let $X=\mathbb{T}^{1} \times \mathbb{R}$. A pinched skew product on $X$ is a map $T: X \rightarrow X$ which can be written as

$$
T(x, y)=(x+\omega, a(x)+b(x) f(x, y))
$$

where $\omega \in \mathbb{R} \backslash \mathbb{Q}, a, b$ and $f$ are continuous functions of their arguments, $f$ is continuously differentiable and bounded, and $b(\hat{x})=0$ for at least one $\hat{x} \in \mathbb{T}^{1}$. Note that since $a$ and $b$ are continuous functions of the compact space $\mathbb{T}^{1}$ they are also bounded, so there exist positive constants $A, B$ and $M$ such that

$$
|a(x)|<A,|b(x)|<B,|f(x, y)|<M
$$

for all $x \in \mathbb{T}^{1}$ and $y \in \mathbb{R}$. The pinch condition is the existence of $\hat{x}$ with $b(\hat{x})=0$ since this implies that $T(\hat{x}, y)=(\hat{x}+\omega, a(\hat{x}))$ for all $y \in \mathbb{R}$.

Example 1. The map (1) is a pinched skew product with $a(x) \equiv 0$ and $b(x)=$ $B \cos 2 \pi x$, so $\hat{x}=\frac{1}{4}$ and $\hat{x}=\frac{3}{4}$ are solutions to the equation $b(x)=0$. Finally, $f(x, y)=\tanh y$ and so $|f(x, y)| \leq 1$, i.e. $f$ is bounded.

Two definitions are needed before the main results can be stated. The idea of global attractors is used widely in problems in high dimensions (e.g. (Hale 2001)), whilst upper semi-continuity and lower semi-continuity are weaker versions of continuity which are used in analysis.

Definition 2. Let $T: X \rightarrow X$ be a pinched skew product. Then the global attractor of $T, \mathcal{A}$, is the set

$$
\mathcal{A}=\bigcap_{n \geq 0} T^{n}(X)
$$

Definition 3. The function $g: \mathbb{T}^{1} \rightarrow \mathbb{R}$ is upper semi-continuous at $x$ if

$$
\limsup _{n \rightarrow \infty} g\left(x_{n}\right) \leq g(x)
$$

for all sequences $\left(x_{k}\right)_{1}^{\infty}$ which tend to $x$. The function $g$ is upper semi-continuous if it is upper semi-continuous at all $x \in \mathbb{T}^{1}$.

Similarly, a function $g$ is lower semi-continuous at $x$ if $-g$ is upper semi-continuous at $x$, and $g$ is lower semi-continuous if $-g$ is upper semi-continuous. 
An intuitive sense of upper semi-continuity can be obtained from the following standard example.

Example 4. Let $I=[-1,1]$ and $A \subset I$. Define the characteristic function of $A, \chi_{A}$, by

$$
\chi_{A}(x)= \begin{cases}1, & \text { if } x \in A \\ 0, & \text { if } x \notin A\end{cases}
$$

Then $A$ is closed if and only if $\chi_{A}$ is upper semi-continuous. Thus the intuitive picture in this zero-one case is that the function is upper semi-continuous if it takes the value zero on a union of open intervals, and 'jumps' upwards at a closed set of points.

With these definitions it is possible to state the main result of this note.

Theorem 5. Let $T: X \rightarrow X$ be a pinched skew product. Then there exists an upper semi-continuous function $\phi: \mathbb{T}^{1} \rightarrow \mathbb{R}$ and a lower semi-continuous function $\psi: \mathbb{T}^{1} \rightarrow \mathbb{R}$ such that the global attractor of $T, \mathcal{A}$, is given by

$$
\mathcal{A}=\{(x, y) \in X \mid \psi(x) \leq y \leq \phi(x)\}
$$

Moreover, $\psi(x)=\phi(x)$ on a dense set of values of $x$.

The sets with graphs $\phi$ and $\psi$ will be referred to as boundary sets, and we will sometimes abuse terminology a little by referring to $\phi$ and $\psi$ as boundary sets rather than graphs of boundary sets. The proof of this result follows the equivalent parts of Keller's results (Keller 1996), and this is given in the next section. In section four some consequences of this result are given and applied to the example of Grebogi et al. (1984).

\section{Proof of Theorem 5}

Let $T: X \rightarrow X$ be a pinched skew product, (3), with bounds (4). Then clearly the second coordinate of $T, T_{2}=\pi_{2} \circ T$ where $\pi_{2}: X \rightarrow \mathbb{R}$ is the projection operator onto the second coordinate, is bounded: $\left|T_{2}(x, y)\right|<A+B M$ for all $(x, y) \in X$. Define $\phi_{1}(x)=A+B M, \psi_{1}(x)=-(A+B M)$ and for each $x \in \mathbb{T}^{1}$ and for each $x \in \mathbb{T}^{1}$ let $I_{1}(x)=\left[\psi_{1}(x), \phi_{1}(x)\right]$. Now define $\phi_{n}, \psi_{n}$ and $I_{n}$ for $n \geq 2$ inductively by

$$
\begin{aligned}
& \phi_{n}(x)=\sup \left\{T_{2}(x-\omega, y) \mid y \in I_{n-1}(x-\omega)\right\} \\
& \psi_{n}(x)=\inf \left\{T_{2}(x-\omega, y) \mid y \in I_{n-1}(x-\omega)\right\}
\end{aligned}
$$

and

$$
I_{n}(x)=\left[\psi_{n}(x), \phi_{n}(x)\right]
$$

Finally, let

$$
\mathcal{A}_{n}=\bigcup_{x \in \mathbb{T}^{1}} I_{n}(x)
$$


A series of simple induction arguments establishes that

(i) $I_{n}(x)$ is a closed interval (possibly trivial);

(ii) $I_{n+1}(x) \subseteq I_{n}(x)$,

(iii) $\phi_{n}(x)$ is a continuous function of $x$;

(iv) $\psi_{n}(x)$ is a continuous function of $x$; and

(v) $\mathcal{A}_{n+1} \subseteq T^{n}(X) \subseteq \mathcal{A}_{n}$.

Now, by definition and property (ii) above,

$$
\psi_{n}(x) \leq \psi_{n+1}(x) \leq \phi_{n+1}(x) \leq \phi_{n}(x)
$$

Thus $\left(\phi_{n}\right)$ is a decreasing sequence of continuous functions, bounded below (by $\psi_{1}$ for example) and hence it tends to a limiting function, $\phi(x)$. Let $\left(x_{k}\right)$ be any sequence converging to $x$ such that $\lim _{k \rightarrow \infty} \phi\left(x_{k}\right)$ exists and equals $\ell$ say. Then for each $n \in \mathbb{N}$

$$
\ell=\lim _{k \rightarrow \infty}\left(\phi_{n}\left(x_{k}\right)-\left[\phi_{n}\left(x_{k}\right)-\phi\left(x_{k}\right)\right]\right)
$$

and since $\phi_{n}(x) \geq \phi(x)$ and $\phi_{n}$ is continuous,

$$
\ell \leq \lim _{k \rightarrow \infty} \phi_{n}\left(x_{k}\right)=\phi_{n}(x)
$$

So, since (15) holds for each $n, \ell \leq \phi(x)$, and hence $\phi$ is upper semi-continuous.

Similarly, $\left(\psi_{n}\right)$ is an increasing sequence of functions bounded above, and hence tends to a limit, $\psi$, which is lower semi-continuous. The characterization of the global attractor, (8), now follows from the definitions of $\phi$ and $\psi$ together with property (v) above.

Finally note that if $x-n \omega=\hat{x}$, where $b(\hat{x})=0$, then $I_{n+1}(x)$ is a single point and $I_{k}(x)$ is a single point for all $k \geq n+1$. At these points (which are dense on the circle) $\psi_{k}(x)=\phi_{k}(x)$ for all $k \geq n$ (and, indeed, is independent of $k$ ), and hence $\psi(x)=\phi(x)$ on a dense set of values of $x$. This completes the proof of Theorem 5 .

Remark 6. The proof of properties (i)-(v) is straightforward, but for completeness we provide a sketch of each argument. It is convenient to refer to the set $\left(x, I_{n}(x)\right)$ as $I_{n}$ to reduce the number of brackets used. Property (i) follows as $I_{n}$ is the image of a closed interval and $T$ is continuous. Property (ii) follows by induction and the fact that $I_{n+1}$ is the image of points in $T^{-1}\left(I_{n}\right)$ whilst $I_{n}$ is the image of points in $T^{-1}\left(I_{n-1}\right)$ (a larger set). Since $T$ is a continuous function of $x$, and maxima and minima of continuous families of continuous functions vary continuously, properties (iii) and (iv) can be established. Finally, $T(X) \subseteq \mathcal{A}_{1}$ implies that $T^{n}(X) \subseteq \mathcal{A}_{n}$, and $\mathcal{A}_{2}=T\left(\mathcal{A}_{1}\right) \subseteq T(X)$ implies that $\mathcal{A}_{n+1} \subseteq T^{n}(X)$ and hence property (v) holds.

\section{Consequences}

Let $P$ denote the set of $x$ which satisfy $b(x)=0$ and for any point $\hat{x} \in P$ let $\operatorname{Orb}(\hat{x})$ denote the orbit of the point $(\hat{x}, y) \in \mathbb{T}^{1}$, i.e. $\operatorname{Orb}(\hat{x})=\left\{T(\hat{x}, y), T^{2}(\hat{x}, y), \ldots\right\}$, which is independent of $y$. Finally, let $c \ell(R)$ denote the closure of a set $R$. Then the detail 
of the dynamics of pinched skew products is closely related to the geometry of the set

$$
\Gamma=\bigcup_{\hat{x} \in P} \operatorname{Orb}(\hat{x})
$$

Note that $\Gamma$ is contained in the intersection of the boundary sets $\phi$ and $\psi$. One of the important properties proved by Keller (1996) for the upper semi-continuous attractor $\Psi$ in the cases he considers is that it has an invariant graph, that is

$$
(x+\omega, \Psi(x+\omega))=T(x, \Psi(x))
$$

or, for the pinch skew products (3)

$$
\Psi(x+\omega)=a(x)+b(x) f(x, \Psi(x))
$$

Unfortunately, the boundary sets $\phi$ and $\psi$ of Theorem 5 are not invariant in general. However, there is one case when they are both continuous and invariant.

Lemma 7. Suppose that the global attractor of a pinched skew product has bounding sets $\phi$ and $\psi$. If $\phi=\psi$ then the resulting set is invariant, continuous and equal to $c \ell(\Gamma)$.

Proof. If $\phi=\psi$ then this set is both upper and lower semi-continuous, which implies that it is continuous (a straightforward exercise) and equal to $\mathcal{A}$ (using Theorem 5) which is invariant by definition. Moreover $\Gamma \subseteq \phi$ and since $\phi$ is continuous and the projection of $\Gamma$ onto the $\mathbb{T}^{1}$ coordinate is dense ( $\omega$ is irrational), $\phi=c \ell(\Gamma)$.

The stability of an invariant curve is determined by the Liapunov exponent in the $y$-direction: let $f_{y}$ denote the partial derivative of $f$ with respect to the second argument, the the Liapunov exponent for typical points on an invariant curve $\vartheta$ is $\lambda_{\vartheta}$ where

$$
\lambda_{\vartheta}=\int_{0}^{1} \log \left|b(x) f_{y}(x, \vartheta(x))\right| d x
$$

If $\lambda_{\vartheta}>0$ then typical points on the curve are unstable.

Lemma 8. Suppose that a pinched skew product has a continuous invariant curve $\vartheta$. Then $\vartheta$ is the only continuous invariant curve of the system, $\vartheta=c \ell(\Gamma)$ and if $\lambda_{\vartheta}>0$ then $\phi \neq \psi$ and at least one of these boundary sets is not continuous.

Proof. If $\vartheta$ is a continuous invariant curve then $\vartheta$ contains all the pinch points and their orbits, so $\vartheta=c \ell(\Gamma)$. This is true of all continuous invariant curves, so $\vartheta$ is unique. If $\lambda_{\vartheta}>0$ then $\vartheta$ cannot be the only set in the global attractor (as it is not stable) and hence $\phi \neq \psi$ and at most one of them equals $\vartheta$ (so at least one of them is not continuous). 
It seems likely that if $\vartheta$ exists and $\lambda_{\vartheta}>0$ then neither $\phi$ nor $\psi$ is continuous, but I have been unable to prove this conjecture in general. See (Sturman and Stark 2000) for results involving Liapunov exponents of the system. Of course, if the pinched skew product is invariant under a symmetry which maps $\phi$ to $\psi$ and vice versa, then either $\phi$ and $\psi$ are both continuous or neither is continuous. This is the case for the original example of Grebogi et al. (1984).

Example 9. Let us return to equation (1). As discussed in the introduction, the circle with $y=0$ is invariant (and continuous), so if $\lambda_{0}$ defined in equation (2) is positive, i.e. if $B>2$, then at least one of the boundary sets $\psi$ or $\phi$ is not continuous. Now, equation (1) is invariant under reflection in the invariant circle: $(x, y) \mapsto(x,-y)$ and hence the upper boundary of $\mathcal{A}$ is mapped to the lower boundary and vice versa by this reflection, i.e. $\psi(x)=-\phi(x)$. Hence neither of the boundary sets have continuous graphs if $B>2$ ( $\phi$ is upper semi-continuous and $\psi$ is lower semi-continuous).

Although the boundary sets $\phi$ and $\psi$ are not invariant in general, there is a special case for which they are invariant.

Lemma 10. Let $T$ be a pinched skew product and suppose that for each $x \in \mathbb{T}^{1}$ $f(x, y)$ is a monotonic function of $y$. Then the union of the boundary sets $\phi$ and $\psi$ is invariant.

Proof. Since the function $f$ is monotonic in the second variable, $b(x) f(x, y)$ is monotonic in $y$ for each fixed $x$. Hence for all $\left(x, y_{1}\right)$ and $\left(x, y_{2}\right)$ in $\mathbb{T}^{1}, y_{1}<y_{2}$ implies that either $T_{2}\left(x, y_{1}\right) \leq T_{2}\left(x, y_{2}\right)$ or $T_{2}\left(x, y_{1}\right) \geq T_{2}\left(x, y_{2}\right)$, depending on the sign of $b(x)$ and whether $f$ is decreasing or increasing. This implies that the end-points of the intervals $I_{n}(x-\omega)$ constructed in the proof of Theorem 5 in section two map to the end points of the intervals $I_{n+1}(x)$, i.e.

$$
\left(x, \phi_{n+1}(x)\right) \cup\left(x, \psi_{n+1}(x)\right)=T\left(x-\omega, \phi_{n}(x-\omega)\right) \cup T\left(x-\omega, \psi_{n}(x-\omega)\right)
$$

and so, in the limit

$$
(x, \phi(x)) \cup(x, \psi(x))=T(x-\omega, \phi(x-\omega)) \cup T(x-\omega, \psi(x-\omega))
$$

Hence the union of the two boundary sets is invariant.

Example 11. Continuing to treat the example of Grebogi et al. (1984), (1), we take $b(x)=B \cos 2 \pi x$ and $f(x, y)=\tanh y$, so $f$ is increasing. Lemma 10 implies that the union of the boundary sets is invariant. In fact, the results of Keller (1996) imply that these sets form the attractor of almost every point in $X$ if $B>2$.

Remark 12. I conjecture that any monotonic pinched skew product has zero topological entropy, and hence that any strange set is nonchaotic. It would be interesting to obtain conditions which would allow Lemma 10 to be strengthened so that the closure of the boundary sets attracts almost all initial conditions. 


\section{Conclusion}

The results described here make it possible to extend some of the work of Keller (1996) to a larger class of maps. These maps all have the skew product structure used to model quasiperiodically forced systems, although the pinching condition cannot be satisfied by maps obtained from continuous time systems, as these must be invertible. Even so, these general results are suggestive, and may give an indication of the techniques and definitions which will prove useful in the study of invertible skew products. The study of pinched skew products is interesting in that some rigorous results are possible, the systems are simple to construct, and they give an indication of the possible behaviour of more general quasiperiodically forced systems. It is worth noting that the existence of stable continuous invariant curves, which has not been a focus of this note, can be explored using the techniques of Stark and Sturman (Stark 1997; Sturman and Stark 2000). This exploits the Liapunov exponent analysis and can be used to show that the conditions of Lemma 7 hold. Those papers, together with this note, provide a useful set of tools with which to study pinched skew products.

We conclude with two questions. First, the attractor $\mathcal{A}$ of Theorem 5 is an example of what economists refer to as a correspondence, i.e. a map from points to sets, see for example, (Herings 1995; Mas-Colell et al. 1995). Are there characterisations of correspondences in the economics literature which would be useful in the description of strange nonchaotic attractors? Second, the results obtained here do not describe the dynamics on the global attractor. It would be interesting to know how to characterise the internal dynamics on this object (for example, when is the dynamics dense in $\mathcal{A}$ ?).

\section{ACKnowledgements}

I am grateful to Demetris Hadjiloucas and Jaroslav Stark for conversations on strange nonchaotic attractors which have influenced this note.

\section{REFERENCES}

Bondeson A., Ott E. \& Antonsen Jr. T.M., 1985, Quasiperiodically forced damped pendula and Schrödinger equations with quasiperiodic potentials: implications of their equivalence. Phys. Rev. Lett., 55, 2103-2106.

Feudel U., Kurths J. and Pikovsky A.S., 1995, Strange non-chaotic attractor in a quasiperiodically forced circle map. Physica D, 88, 176-186.

Grebogi C., Ott E., Pelikan S. and Yorke J.A., 1984, Strange attractors that are not chaotic. Physica D, 13, 261-268.

Hale J.K., 2001, Attractors and dynamics in partial differential equations. In From finite to infinite dimensional dinamical systems, edited by J.C. Robinson and P.A. Glendinning (Kluwer NATO Series II. 19, Dordrecht), pp.85-113. 
Herings P.J-J., 1995, Equilibrium existence results for economies with price rigidities. Economic Theory, 7, 63-80.

Keller G., 1996, A note on strange nonchaotic attractors. Fundamenta Mathematicae, 151, 139-148.

Mas-Colell A., Whiston, M.D. and Green J.R., 1995, Microeconomic Theory (Oxford University Press, New York).

Pikovsky A. and Feudel U., 1994, Characterizing strange nonchaotic attractors. Chaos, 5, 253-260.

Romeiras F.J. \& and Ott E., 1987, Strange nonchaotic attractors of the damped pendulum with quasiperiodic forcing. Phys. Rev. A, 35, 4404-4413.

Stark J., 1997, Invariant graphs for forced systems. Physica D, 109, 163-179.

Sturman R. and Stark J., 2000, Semi-uniform ergodic theorems and applications to forced systems. Nonlinearity, 13, 113-143.

Department of Mathematics, UMist, P.O. Box 88, Manchester M60 1QD, United Kingdom. E-MAil: P.A.Glendinning@Umist.AC.uK 